\title{
Two Cases of Esophageal Injury Following Anterior Cervical Discectomy and Fusion: One Overt and One Covert
}

\author{
Prasad Krishnan $^{a} \quad$ Rajaraman Kartikueyan ${ }^{a} \quad$ Sachinkumar Maheshbhai Patel ${ }^{a}$

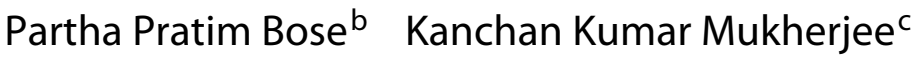 \\ ${ }^{a}$ Department of Neurosurgery, National Neurosciences Centre, and ${ }^{b}$ Department of Gastromedicine, \\ Peerless Hospital, Kolkata, and 'Department of Neurosurgery, PGIMER, Chandigarh, India
}

\section{Keywords}

Anterior cervical spinal discectomy · Esophageal

injury · Dysphagia - Mediastinitis - Cervical osteomyelitis ·

Esophagography

\begin{abstract}
Esophageal perforation is a dreaded complication of anterior cervical spinal surgery. A 52-year-old diabetic man had undergone a surgery for a C6-C7 disc prolapse and developed spiking fever with chills and rigor on the 7th postoperative day. No cause could be found out but a CT scan of thorax done in the course of investigations revealed pneumomediastimum. The patient succumbed on the 10th day after surgery. Autopsy revealed the cause of death to be mediastinitis following iatrogenic esophageal perforation. A second patient, 53 years of age, following surgery for $\mathrm{C} 5-\mathrm{C} 6$ disc prolapse and developed intractable dysphagia. Later, fever and purulent discharge from the wound prompted an MRI showing prevertebral collection extending to the superior mediastinum. Presuming only wound infection, debridement and implant removal was done. However persistent serous discharge from the wound revealed an esophageal injury. Late diagnosis precluded primary repair. With conservative treatment, the fistula finally closed after 42
\end{abstract}

days. Postoperative dysphagia, a common complaint following surgery, may not always be present in cases of esophageal injury. A high index of suspicion is required for diagnosing and initiating treatment for esophageal perforation before complications set in.

(c) 2017 S. Karger AG, Base

\section{Introduction}

Anterior cervical discectomy and fusion (ACDF) is routinely performed for the treatment of cervical myelopathy or radiculopathy as well as in some cases of cervical spinal injury [1]. While generally a safe procedure, complications include trauma to neural structures (spinal cord or roots), graft or implant failure, injury to recurrent laryngeal nerve and neck vessels, and rarely esophageal injury [2]. The incidence of esophageal injury is reported to vary between 0.02 and $1.52 \%$, with a mortality rate of upto $19 \%$ [1]. Fountas et al. [3] have observed that such complications are often under-reported and comment that "their exact incidence in large clinical series is lacking." In their retrospective analysis of 1,015 patients, 3 had esophageal perforation (of whom 1 died).

\section{KARGER}

(c) 2017 S. Karger AG, Basel

E-Mail karger@karger.com

www.karger.com/aon
Prasad Krishnan

Department of Neurosurgery, National Neurosciences Centre

Peerless Hospital Campus, 2nd Floor

360 Panchasayar, Kolkata 700094 West Bengal (India)

E-Mail prasad.krishnan@ rediffmail.com 

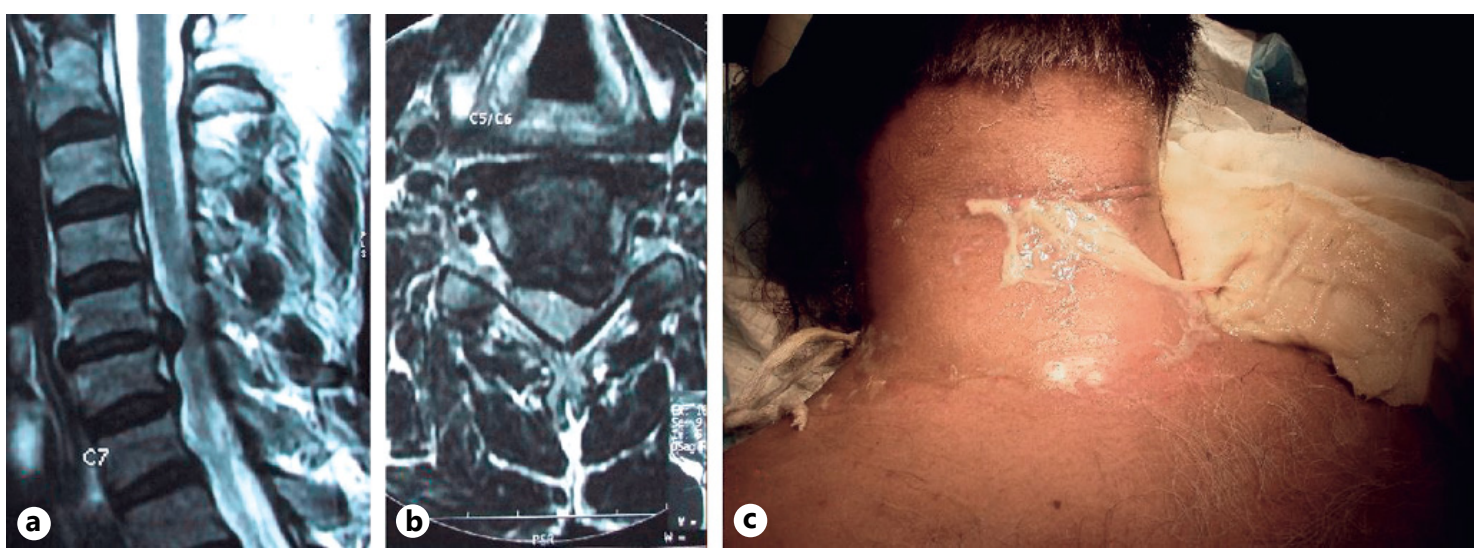

Fig. 1. Sagittal T2 (a) and axial MRI sequence (b) showing C5-C6 left sided disc prolapse; clinical photograph (c) showing pus discharge from the lateral side of the incision.

Fig. 2. a Chest X-ray showing bilateral lower zone haziness suggestive of aspiration pneumonitis; b sagittal T2 MRI sequence showing implants in situ and large prevertebral collection extending upto D3 vertebral body level.

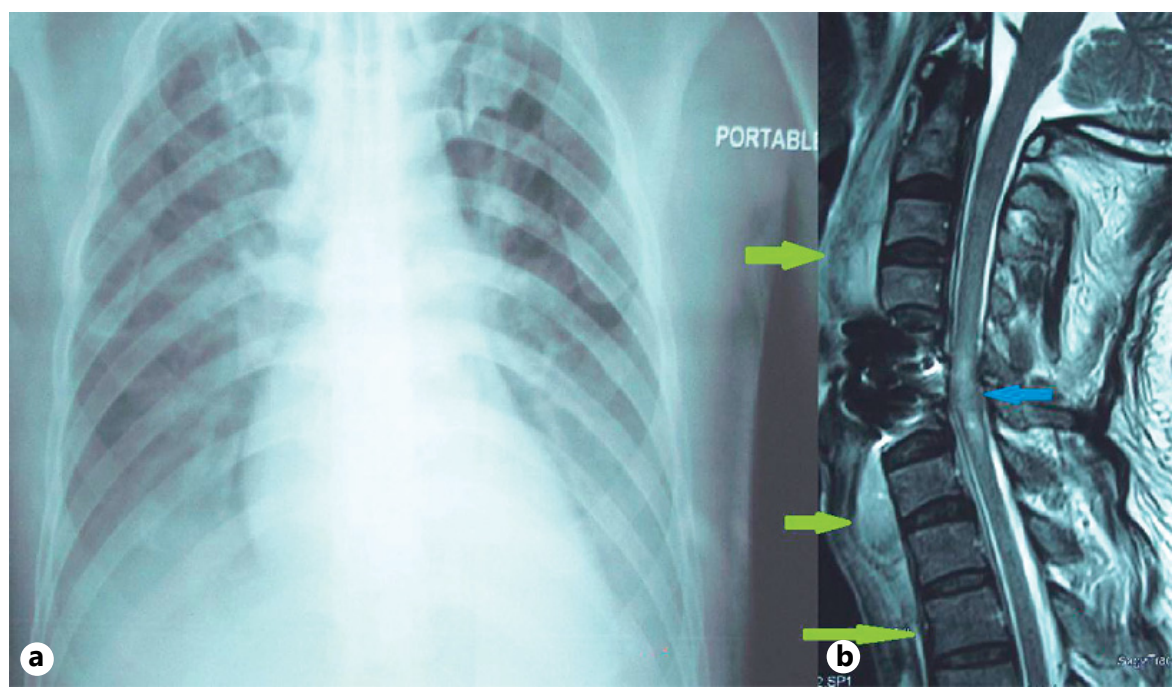

\section{Case Report}

A 52-year-old diabetic male patient underwent C6-C7 ACDF for cervical radiculopathy (by the senior author K.K.M.) 15 years ago. Postoperatively he had no dysphagia and complete relief of his radicular pain. On the 7th postoperative day, he developed fever with chills and rigor and also became hypotensive with decreasing urine output and required ionotropic support. Blood investigations revealed high total counts and C-reactive protein (CRP) levels. No obvious focus of infection could be discovered. Empirical antibiotics were instituted to no avail. A CT scan of chest done on the 9th postoperative day revealed air in the mediastinum. He died the following day and autopsy revealed the cause of death to be mediastinitis following esophageal perforation.

A 53-year-old gentleman underwent ACDF, 1 year ago at another centre, for a C5-C6 prolapsed intervertebral disc (Fig. 1a, b) causing left sided brachialgia and neck pain. Postoperatively there was neurological deterioration, respiratory difficulty, and dysphagia. Seven days later when shifted to our hospital, power in both upper limbs was grade $3 / 5$. Bilateral hand grip was not formed. Power was grade $3 / 5$ in the right and grade $0 / 5$ in the left lower limb. Plantars were non-elicitable but sensory examination was normal. $\mathrm{He}$ was febrile with wound breakdown and had gross discharge of pus from the neck incision (Fig. 1c). His total counts and CRP levels were high. Chest X-ray showed opacities in the right lower zone suggestive of aspiration (Fig. 2a). MRI of cervical spine showed extensive collection in prevertebral space extending to superior mediastinum with signal changes in the cord and vertebrae at the operated site (Fig. 2b). Suspecting postoperative spondylodisciitis and cervical abscess, the wound was re-explored and pus was drained. Implants were removed and a halo device was used to immobilize the spine. The lateral part of the wound was left open with a corrugated rubber drain extending into the mediastinum. Complete closure of the wound was planned as a secondary procedure once the infection subsided with along with posterior instrumentation to stabilize the spine. The pus grew Klebsiella and the patient was put on injectable antibiotics. Over the next 3 days, the nature of the discharge changed from purulent to serous and watery but the quantity increased. Due to persistent dysphagia and inability to swallow even saliva, esophageal injury was suspected. Gastrografin study revealed peri-esopha- 
Fig. 3. a Esophagography with gastrograffin showing hold up of dye in the esophagus with periesophageal spillage; b CT scan showing large abscess cavity filled with air abutting the carotid sheath and crossing the midline precluding placement of a sternomastoid flap for fear of blocking the drainage of dependant segment.
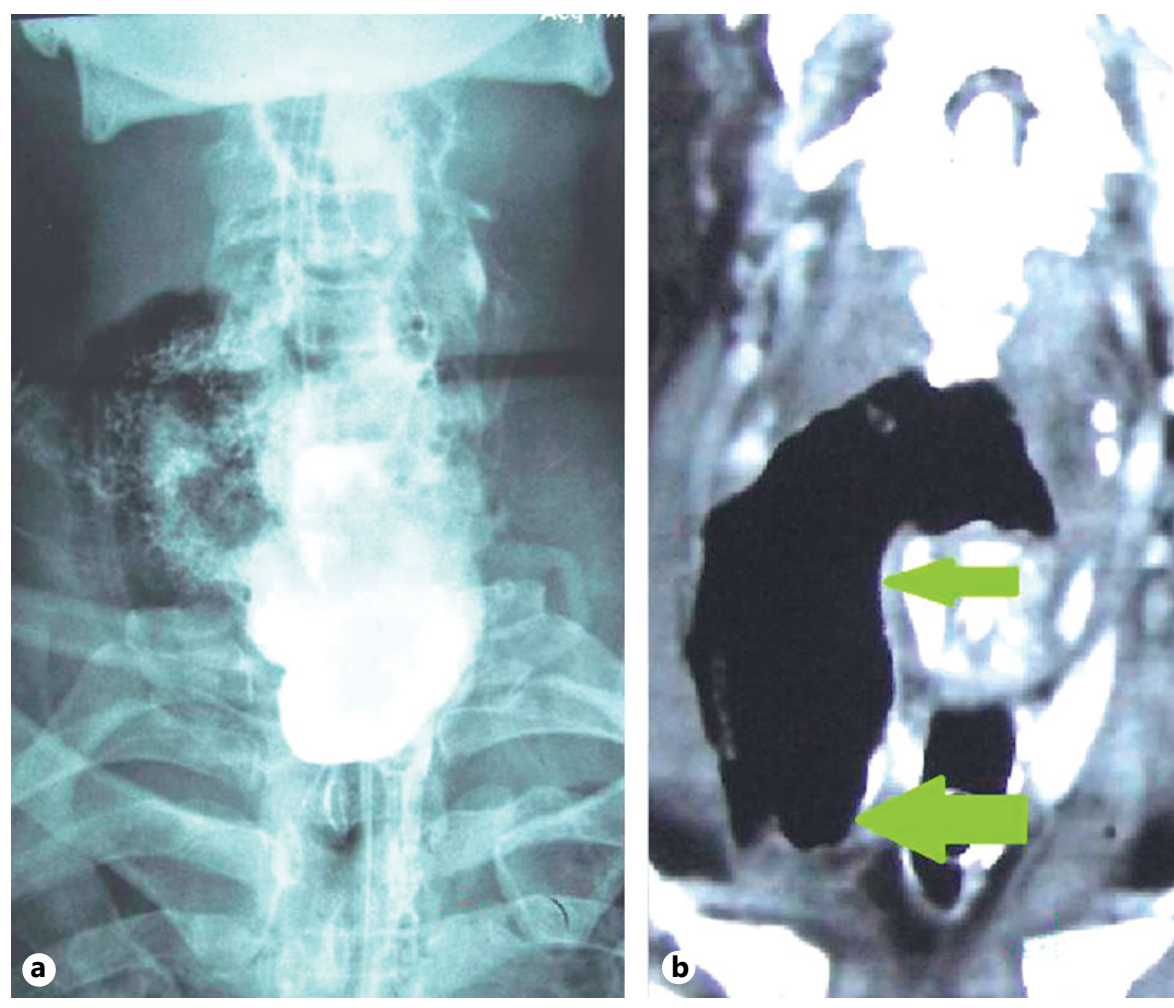

geal spillage of dye with holdup of the contrast in upper part of the esophagus (Fig. 3a). A primary repair of the esophagus was ruled out given the amount of inflammation, and due to active infection and large residual abscess cavity on CT scan (Fig. 3b), using muscle flap closure (sternocliedomastoid) was also deferred. Esophagoscopy was done but could not show the location of the rent and due to spasm, the scope could not be passed distally. Furthermore, the leak was deemed to be too high to place a self expanding metallic stent. A feeding jejunostomy was then done to maintain nutrition. With regular irrigation and dressing, over 4 weeks, the wound discharge subsided and the opening became progressively narrow and healed by secondary intention in 7 weeks (Fig. 4a). The patient began to swallow saliva and later was put on oral diet as well. His power improved and he became independently ambulant but grip weakness persisted. After 3 months, the vertebrae had fused completely and his halo fixator was removed (Fig. 4b).

\section{Discussion}

Esophageal injuries identified within 1 month of surgery are classified as "early" and called "late" if they come to light afterward [4]. Analyzing the data from 10,000 patients of the Cervical Spine Research Society, Newhouse et al. [5] identified 22 cases of which 6 were detected at the time of surgery and the others were detected later. Injury by sharp instrumentation, esophageal manipulation or vigorous retraction [6], pointed retractor blades [7], or use of cautery may cause intraoperative damage. Revision surgery in the presence of adhesions is another risk factor [7]. Delayed perforations on the other hand are consequent to graft extrusion [6], implant backout [6] or malposition [1], or pressure necrosis secondary to chronic friction of the esophagus during swallowing [2].

The commonest presenting symptom, following intraoperative esophageal injury is dysphagia. This was present in one of our cases but not in the other. However, this is a non-specific complaint as transient postoperative swallowing difficulties are found in $28-57 \%$ of patients [6]. Choking, aspiration, cough, fever, subcutaneous emphysema, or wound leakage $[1,7-9]$ are other ominous features that must alert the surgeon to this possibility. As the pooled secretions carry a risk of mediastinitis, vessel erosion, spondylodiscitis, cervical abscess, and perforations must be treated early and aggressively [2].

Diagnosis can be clinched by a combination of X-rays (showing implant back out, air in the subcutaneous space), MRI or CT scans (showing prevertebral collections and vertebral infection), and esophagoscopy or esophagography (showing the rent or spillage of dye in the adjacent tissues).

Perforation treatment depends on the time of detection [7]. If evident during surgery, primary repair of the rent will suffice. In the early postoperative period, suturing with placement of drain to prevent any collection may suf- 
Fig. 4. a Clinical photograph showing fistulous site healed by secondary intention (green arrows) and medial part of the wound healed by primary intention (blue arrows); b lateral X-ray cervical spine showing bony fusion at C5 and C6.
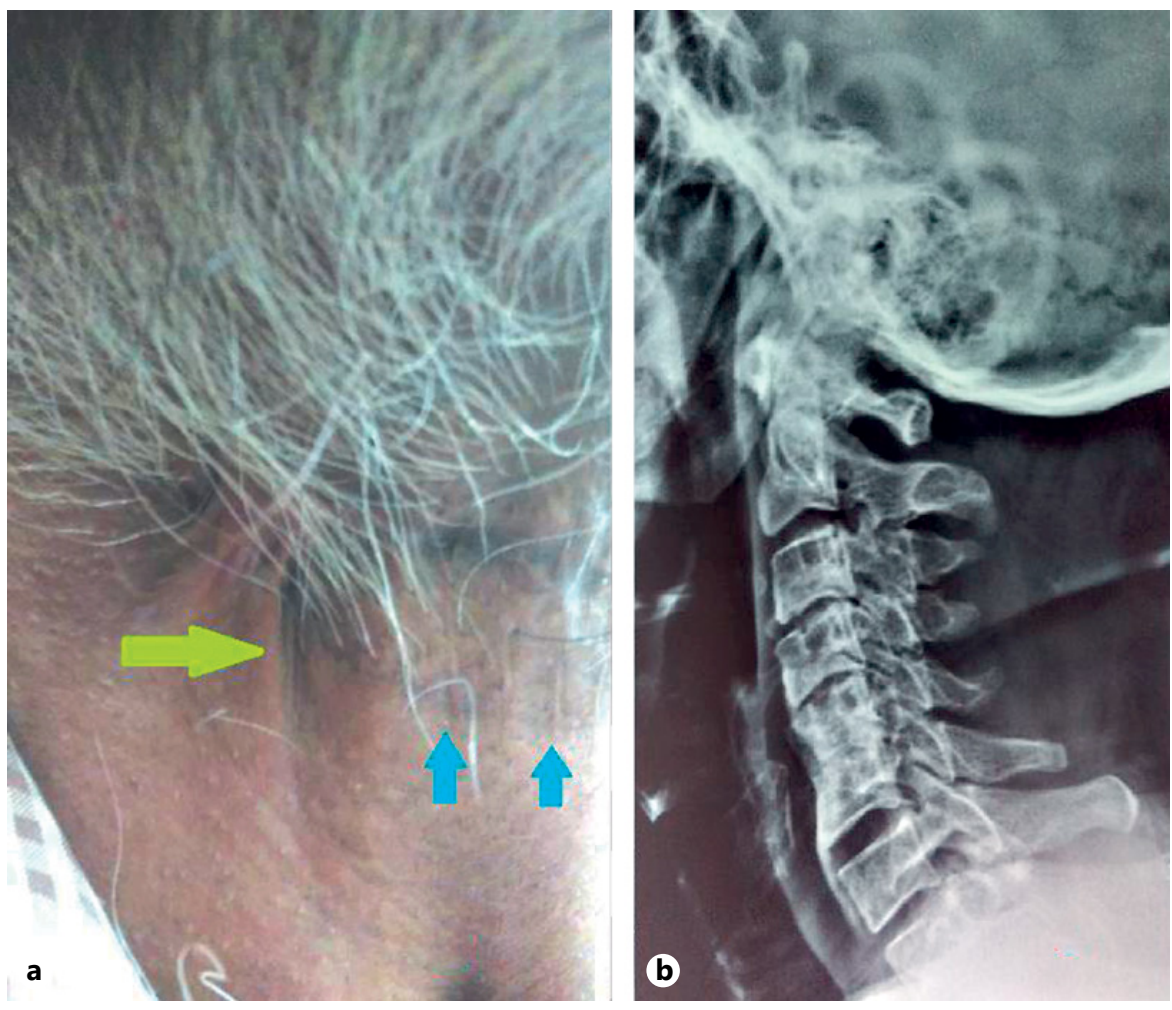

fice [7]. Large rents may require augmentation with muscle [8] or omentum [9]. In late perforations, vascularized muscle flaps (commonly sternocliedomastoid that is available near the operative field [8]) are used to provide a barrier between the esophagus and the spine and can provide blood supply for both healing and antibiotic delivery to the operative site [1]. Spontaneous healing is also known but may take 1-3 months but is said to have a higher rate (20-45\%) of abscess formation [6].

In our first patient, there was no postoperative dysphagia or wound site complications. The first manifestation of injury was delayed postoperative fever. Esophageal injury was a cause and the diagnosis was made only postmortem. In the second patient, despite postoperative dysphagia and respiratory difficulty consequent to aspiration, the injury was recognized late. Though we had this complication in mind, due to the purulent nature of his discharge, we had initially diagnosed him to have postoperative infection with a prevertebral abscess. The perforation in our patient occurred following surgery at the C5-C6 level described in literature as the site of maximal risk [7].

Due to the delayed diagnosis, the tissues were inflamed, infection had set in, and a large dead space was present inferiorly. Hence, no definitive treatment of the perforation by either primary suturing (as the sutures would not hold) or by flap transposition (as a part of the dead space inferiorly might remain loculated and without path for egress of secretions and cause mediastinitis) could be done. Conservative treatment (immobilizing the spine, wound irrigation and dressing, maintaining nutrition, and treating infection with antibiotics) was successful only after a protracted course.

\section{Conclusion}

Esophageal perforation is an uncommonly encountered complication that many surgeons never see. It may be fatal due to risk of mediastinitis, great vessel erosion, or sepsis. It must be borne in mind when dealing with a case of unexplained fever, persistent dysphagia, aspiration, or wound complications after ACDF. Early diagnosis before abscess formation or bony infection will enable primary repair of the esophagus, prevent spinal destabilization secondary to implant removal, and minimize costs and hospital stay for the patient.

\section{Disclosure Statement}

Authors declared no conflict of interest. The patient has consented to submission of this case report to the journal. There is no source of funding. 


\section{References}

1 Amhaz HH, Kuo R, Vaidya R, Orlewicz MS: Esophageal perforation following cervical spine surgery: a review with considerations in airway management. Int J Crit Illn Inj Sci 2013;3:276-278.

2 Yang S, Lee SB, Cho KS: Delayed esophagus perforation after anterior cervical spine surgery. Korean J Neurotrauma 2015;11:191-194.

3 Fountas KN, Kapsalaki EZ, Nikolakakos LG, Smisson HF, Johnston KW, Grigorian AA, Lee GP, Robinson JS Jr: Anterior cervical discectomy and fusion associated complications. Spine (Phila Pa 1976) 2007;32:2310-2317.
4 Harman F, Kaptanoglu E, Hasturk AE: Esophageal perforation after anterior cervical surgery: a review of the literature for over half a century with a demonstrative case and a proposed novel algorithm. Eur Spine J 2016; 25:2037-2049.

5 Newhouse KE, Lindsey RW, Clark CR, Lieponis J, Murphy MJ: Esophageal perforation following anterior cervical spine surgery. Spine (Phila Pa 1976) 1989;14:1051-1053.

6 Nathani A, Weber AE, Wahlquist TC, Graziano GP, Park P, Patel RD: Delayed presentation of pharyngeal erosion after anterior cervical discectomy and fusion. Case Rep Orthop 2015;2015:173687.
7 Yılmaz C, Kabataş S, Gülsen S, Coven I, Caner $\mathrm{H}$ : Esophageal perforation due to revision of anterior cervical spine surgery. Eur J Gen Med 2010;7:210-212.

8 Ahn SH, Lee SH, Kim ES, Eoh W: Successful repair of esophageal perforation after anterior cervical fusion for cervical spine fracture. J Clin Neurosci 2011;18:1374-1380.

9 Hanwright PJ, Purnell CA, Dumanian GA: Flap reconstruction for esophageal perforation complicating anterior cervical spinal fusion: an 18-year experience. Plast Reconstr Surg Glob Open 2015;3:e400. 